\title{
A cooperator-assisted wireless body area network for real-time vital data collection

\author{
Miyu Momoda ${ }^{1 *}$ and Shinsuke Hara ${ }^{2}$
}

\begin{abstract}
Wearable wireless body area network (WBAN) has attracted considerable attention as a means to collect vital data in sports training and urgent life-critical scenarios, which requires high reliability in links from sensor nodes to a central coordinator. Herein, cooperative relaying, which means retransmission not by sensor nodes themselves but through their cooperators, works effectively, since human postures do not suddenly change the states of the links in quite a short duration such as a retransmission interval.

In this paper, we propose a cooperator-assisted WBAN for real-time vital data collection. We show that the WBAN is realizable based on two kinds of medium access control (MAC) protocols such as a hybrid-time divison multiple access/carrier sense multiple access (TDMA/CSMA) and a TDMA, both of which are compliant with the IEEE 802.15.4.e standard, and evaluate the packet error rates and power consumptions for a hybrid-TDMA/CSMA-based WBAN and a TDMA-based WBAN. In the evaluation of packet error rates, we use the stored received signal strength indication (RSSI) data, which were obtained from experiments with three subjects in a realistic scenario composed of a series of different actions and postures in a mixed-indoor/outdoor environment.
\end{abstract}

Keywords: WBAN; Cooperative transmission; CSMA; TDMA

\section{Introduction}

Wireless body area network (WBAN) is a short-range network for connecting nodes on a human body (wearable WBAN) or in a human body (implant WBAN). One key application includes vital data collection for patients and elderly people in medical and health care scenarios, where the energy consumption of sensor nodes is the most important factor for their long-term monitoring.

Another key application is vital data collection for workers such as firefighters, soldiers, and police officers in an urgent/life-critical scenario and for athletes in a sports-training scenario. For the former scenario, it is essential to monitor in real-time their physical and physiological states through collecting vital data from sensor nodes: body temperature meter, electro-cardio-graph (ECG), electro-myo-graph (EMG), tri-accelerometer, and $\mathrm{SpO} 2$ meter (for oxygen saturation) put on various positions of their bodies: left and right arms, left and right

\footnotetext{
*Correspondence: miyu@c.info.eng.osaka-cu.ac.jp

1 Department of Electronic Information System, Graduate School of Engineering, Osaka City University, 3-3-138, Sugimoto, Sumiyoshi, 558-8585

Osaka, Japan

Full list of author information is available at the end of the article
}

ankles, chest, finger and so on. On the other hand, for the latter scenario, it is also important to collect vital data in real-time, since trainers can train athletes with feedback information on their physical states. For the application, high reliability such as low packet error rate and low packet delay is the most important factor, in other words, because of their short operating times, the energy consumption of sensor nodes is not so critical. In this paper, we pay attention to how to guarantee high reliability in a WBAN.

Because of the short transmittable range, typically, less than $2 \mathrm{~m}$, and the low transmission power, typically, less than $0.1 \mathrm{~mW}$, a star topology has been mainly considered in WBANs, connecting a central WBAN coordinator (BANCO) to sensor nodes put on different positions of a human body [1]. For WBANs, candidates in unlicensed frequency bands are the $2.4 \mathrm{GHz}$ industrial, scientific and medical (ISM) band and the $3.1-10.6 \mathrm{GHz}$ ultra wide band (UWB) band, and their propagation channel characteristics have been well investigated [2-4]. In this paper, we assume the $2.4 \mathrm{GHz}-\mathrm{ISM}$ band, because in the frequency band, not only the IEEE 802.15.6 standard is now

\section{Springer}

(C) 2015 Momoda and Hara. Open Access This article is distributed under the terms of the Creative Commons Attribution 4.0 International License (http://creativecommons.org/licenses/by/4.0/), which permits unrestricted use, distribution, and reproduction in any medium, provided you give appropriate credit to the original author(s) and the source, provide a link to the Creative Commons license, and indicate if changes were made. 
available but also a variety of inexpensive transceiver modules have been on the market, which are applicable for WBAN applications $[5,6]$.

Although the 2.4 GHz-ISM band has the advantages mentioned above, its major disadvantage is that it is not rich in diffraction. In a WBAN where there are BANCO and sensor nodes on different positions of a human body, in addition to the poorness of diffraction and the low transmission power, the directivity of antenna is much affected by the human body, so the direct link between them is easily and severely blocked, resulting in link disconnection.

In order to improve the reliability of wireless transmission, it is essential to use "the broadcast nature" inherent to wireless. In this sense, WBAN is advantageous, because there are many sensor nodes which cannot help receiving signals transmitted from other sensor nodes to a BANCO and can act as cooperators for them. It is well known that cooperative transmission, which means the use of cooperators or relays, improves the quality of wireless transmission by means of transmit diversity $[7,8]$. In fact, for WBANs in the $2.4 \mathrm{GHz}-\mathrm{ISM}$ band, the performance of a cooperative transmission is discussed in an indoor walking scenario [9], an opportunistic relaying and a cooperative network coding are proposed [10,11], and the effects of selection combining and maximum ratio combining with the help of relay are examined [12]. In addition, for WBANs assuming the IEEE 802.15.4 standard in the same frequency band [13], the advantage of relaying is suggested [14], and the performance is investigated $[15,16]$. These works show the effectivity of cooperative transmission in WBANs, but their targets are all to improve the energy efficiency for prolonging the lifetime of the networks, which are common to those in general wireless sensor networks. Furthermore, for a sensor node in a WBAN, other sensor nodes can be candidates for its cooperator, so a BANCO needs to select the best one out of them, but these works use a pre-determined sensor node as a cooperator for each sensor node, without discussing how to select it out of them. There are some works addressing cooperator selection schemes, but a single human motion is assumed for a prediction-based relay selection in the 2.4 GHz-ISM band [17], and the UWB band is targeted [18], whose radio propagation characteristic is totally different from that of the $2.4 \mathrm{GHz}-\mathrm{ISM}$ band.

This paper proposes a cooperator-assisted WBAN for ensuring real-time vital data collection $[19,20]$. In a WBAN, the radio channel characteristic around the wearer dynamically changes according to not only his/her action and posture but also the environment surrounding him/her such as indoors and outdoors. This implies that a cooperator suited for each sensor node also dynamically changes according to the physical situation around the wearer, so adaptive cooperator selection recognizing the temporal radio channel characteristic seems interesting and promising. It must be difficult, however, since the physical situations are infinite, so the proposed WBAN selects a single sensor node out of other sensor nodes for each sensor node, which can act as a moderately good cooperator commonly in different physical situations. Furthermore, the proposed cooperator selection is distributed, and in addition to it, the proposed WBAN selects an adequate sensor node as a BANCO. In the performance evaluation of the proposed WBAN, on the other hand, we use the stored received signal strength indication (RSSI) data obtained from experiments with three subjects in a realistic scenario composed of a series of different actions and postures in a mixed-indoor/outdoor environment.

It is better to realize a cooperator-assisted WBAN using a current wireless communication standard, and in this sense, one way is the use of a decode-and-forward transmission in which cooperators retransmit their oncestored packets after their parent sensor nodes transmit packets to a BANCO. Regarding medium access control (MAC) protocol, this is realizable in time division multiple access (TDMA) or carrier sense multiple access (CSMA). TDMA seems suitable for highly reliable cooperatorassisted WBAN, since it can avoid packet collisions. However, TDMA have some problems. One is the lack of frames, namely, selecting one coordinator per sensor node doubles the number of required frames. For instance, in the IEEE 802.15.4-2011 standard, the number of frames per superframe is limited up to 16 including a beacon frame, so this means that the maximum number of sensor nodes is limited up to 7 in a WBAN, which is too small. Another is the delay of retransmission. For successful reception of each frame in a superframe, a BANCO can broadcast its acknowledgement (ACK) packet only in the beacon frame of the next superframe. Therefore, if cooperators retransmit their packets after recognizing their ACKs, the retransmission is delayed by the time period of one superframe.

In this paper, we propose the use of low latency deterministic network (LLDN) in the IEEE 802.15.4e standard [21] for realizing a cooperator-assisted WBAN [22], where we show two superframe designs using a TDMA and a hybrid-TDMA/CSMA. Furthermore, these two designs introduce different power consumptions, so using the values of power consumptions for a typical transceiver module, we compare the power consumptions between them.

The remainder of this paper is organized as follows. Section 2 presents the system model and assumptions. Section 3 describes the superframe design criteria. Section 4 discusses the principle of the cooperator selection and explains the selection algorithms for cooperator 
and BANCO in detail. Section 5 outlines the experiment on the RSSI measurement, and Section 6 shows the performance of the proposed cooperator/BANCO selections using the measured RSSI data. Finally, Section 7 concludes the paper.

\section{System Model and Assumptions}

We assume a WBAN system model that consists of total $N_{\text {node }}$ nodes including vital sensor nodes and one BANCO on a human body. Putting different ID numbers of $1,2, \cdots, N_{\text {node }}$ to the nodes, we define a set of node ID numbers as $\mathrm{V}_{\text {node }}$. We also assume that any node can measure the RSSI for its received packet. Each node periodically senses vital signs and transmits its data packets to a BANCO, and then, the BANCO transmits collected data to an off-body node for monitoring his/her physical and physiological state. The purpose of this paper is to improve the reliability of the wireless network among the sensor nodes and the BANCO. Thus, the network between the BANCO and the off-body node is out of the scope of the paper. For example, we can use $3 \mathrm{G} / \mathrm{LTE}$ as the network [23].

\subsection{Cooperative transmission}

Figure 1 shows the system model on a cooperator-assisted WBAN, where a source node (A) transmits packets to a BANCO (B) with a cooperator (C). According to the promiscuous mode operation in all nodes, $C$ can receive and store packets transmitted from $\mathrm{A}$ to $\mathrm{B}$. In the hybrid TDMA/CSMA, when A transmits a packet to $\mathrm{B}$, if $\mathrm{C}$ can recognize the failure of the packet transmission, $C$ transmits the stored packet instead of A, otherwise, C does not transmit it. In the TDMA, on the other hand, regardless of whether the packet transmission from $A$ to $\mathrm{B}$ fails or not, $\mathrm{C}$ always transmits the stored packet to $\mathrm{B}$.

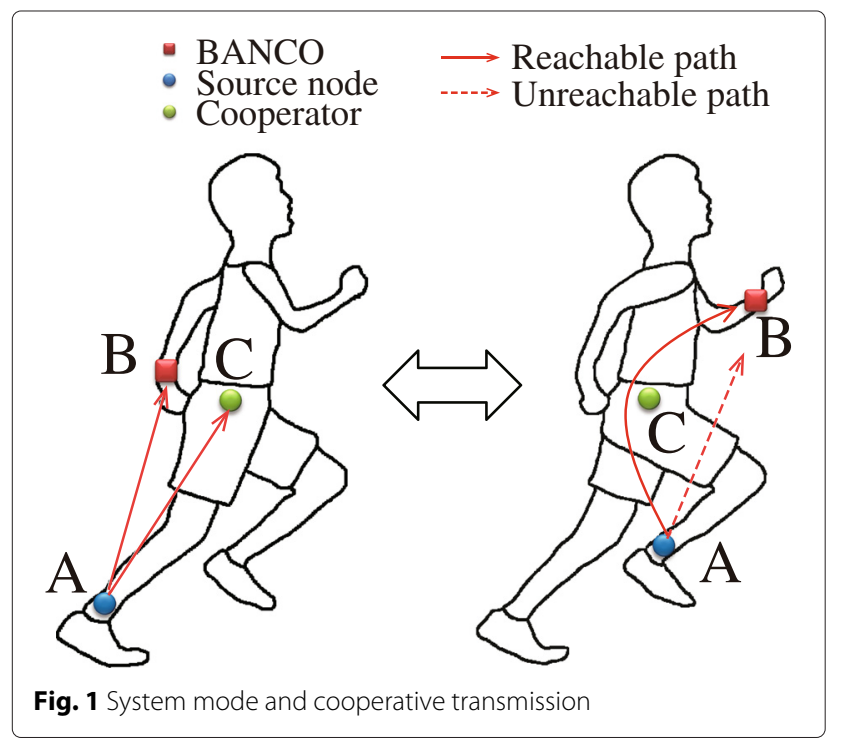

Hereafter, we define the indices for the source node, cooperator candidate and temporarily selected BANCO as $i\left(i=1,2, \cdots, N_{\text {node }}\right), j\left(j=1,2, \cdots, N_{\text {node }}, j \neq i, j \neq k\right)$, and $k\left(k=1,2, \cdots, N_{\text {node }}, k \neq i\right)$, respectively.

\subsection{Sensor node model}

We assume that a node has $N_{\text {sensor }}$ kinds of sensors all operating with analog to digital (A/D) resolution of $q$ bits/sample and sampling rate of $F \mathrm{~Hz}$, and it aggregates $M_{\mathrm{s}}$ successively sensed data into a single data burst with additional information of $d$ bits such as timestamp. Therefore, the payload size is given by

$$
L_{\text {payload }}=\left(q \times N_{\text {sensor }}+d\right) \times M_{\mathrm{s}} \text { bits. }
$$

Then, the node assembles a data packet by adding an overhead with length of $L_{0}$ bits to the payload. Defining the data transmission rate as $R$ bits/s, the data packet duration becomes

$$
T_{\mathrm{d} a t a}=\left(L_{\mathrm{p} a y l o a d}+L_{\mathrm{o}}\right) / R \text { sec. }
$$

\subsection{Packet success rate model}

The fading channel characteristic dynamically changes according to the actions of the BAN wearer, but because of the short duration of data packet, it is reasonable to assume that each data packet experiences "block fading," which means that the fading channel characteristic is constant over the duration of the packet. In this case, the packet success rate for the $n$th packet $\left(n=1,2, \cdots, N_{\text {packet }}\right)$ from the $l$ th node to the $m$ th node $\left(l, m=1,2, \cdots, N_{\text {node }}, l \neq m\right)$ is given by

$$
\operatorname{PSR}_{l, m}(n)=\left(1-\left(1-B E R_{l, m}(n)\right)^{L_{\text {packet }}}\right)
$$

where $L_{\text {packet }}$ and $B E R_{l, m}(n)$ are the packet length in bits and the bit error rate in the $n$th packet from the $l$ th node to the $m$ th node, respectively. We refer to the IEEE 802.15.4 standard [13] as the bit error rate:

$$
B E R_{l, m}(n)=\frac{8}{15} \times \frac{1}{16} \times \sum_{\lambda=2}^{16}(-1)^{\lambda}\left(\begin{array}{c}
16 \\
\lambda
\end{array}\right) e^{20 \times S N R_{l, m}(n) \times\left(\frac{1}{\lambda}-1\right)}
$$

where $S N R_{l, m}(n)$ is the signal to noise power ratio (SNR) for the $n$th packet from the $l$ th node to the $m$ th node:

$$
\operatorname{SNR}_{l, m}(n)=\operatorname{RSSI}_{l, m}(n) / P_{\text {noise }} .
$$

In (5), $\operatorname{RSSI}_{l, m}(n)$ is the measured RSSI for the $n$th packet from the $l$ th node to the $m$ th node, and $P_{\text {noise }}$ is the noise power given by

$$
P_{\text {noise }}=N F+N_{\text {noise }}+10 \log _{10} B_{\text {ch }} \mathrm{dBm}
$$

where $N F, N_{\text {noise }}$ and $B_{\mathrm{ch}}$ are the noise figure, thermal noise density, and channel bandwidth, respectively. 


\section{Superframe design criteria}

The IEEE 802.15.4e standard defines the LLDN that is described as follows: a superframe is divided into a beacon slot and timeslots of equal length which are assigned to specific nodes. A coordinator periodically transmits beacons for synchronization with the superframe structure. Each timeslot is able to be assigned to exactly one node, called a slot owner, which has access privilege in the timeslot, namely, it transmits without using CSMA. Provided that more than one node wishes to transmit in a timeslot, which is referred to as a shared group timeslot, any nodes except a coordinator transmit using CSMA. In a shared group time slot, a coordinator broadcasts a clear to send (CTS) shared group frame to indicate that the timeslot is not used by the slot owner. Then, a node sends a request to send (RTS) frame and waits for a CTS frame from the coordinator in a response to it. After data transmission of other nodes, the coordinator can fully or partly occupy a timeslot for data transmission as well.

In the following two subsections, we show how to apply the LLDN to the cooperator-assisted BAN system.

\subsection{LLDN-based hybrid TDMA/CSMA}

As shown in Fig. 2a, a superframe is divided into $N_{\text {node }}-1$ shared group timeslots. The slot owner of the $i$ th timeslot is exactly the $i$ th node, and it selects its cooperator as a member of its shared group. In the $i$ th timeslot, the $i$ th node is able to send its packet to a BANCO without packet collision, namely, in a TDMA manner. Only if the BANCO does not successfully receive the packet, it transmits a CTS shared group frame back to the $i$ th node, so the $i$ th cooperator attempts to transmit its stored packet. Here, the key is that the CTS shared group frame acts as a negative acknowledgement (NACK) packet for the ith node as well as its cooperator.

Defining the durations of beacon, clear channel assessment (CCA), short interframe space (SIFS), timeout time, long interframe space (LIFS), backoff time, CTS shared group frame, CTS frame and RTS frame as $T_{\text {beacon }}, T_{\mathrm{CCA}}$, $T_{\mathrm{SIFS}}, T_{\mathrm{t} o}, T_{\mathrm{LIFS}}, T_{\mathrm{b} o}, T_{\mathrm{CTSSG}}, T_{\mathrm{CTS}}$, and $T_{\mathrm{R} T S}$, respectively, in order to accommodate $\left(N_{\text {node }}-1\right)$ timeslots within a single superframe with duration of $T_{\mathrm{S} F}$, the time slot duration $T_{\mathrm{ts}}$ needs to satisfy the following two criteria:

$$
\begin{aligned}
T_{\mathrm{SF}}= & M_{\mathrm{S}} / F \geq T_{\mathrm{t} s} \times\left(N_{\mathrm{node}}-1\right)+T_{\mathrm{beacon}}+T_{\mathrm{SIFS}}(7) \\
T_{\mathrm{t} s} \geq & T_{\mathrm{t} o}+T_{\mathrm{CTSSG}}+T_{\mathrm{b} o}+T_{\mathrm{CCA}}+T_{\mathrm{R} T S} \\
& +2 \times T_{\mathrm{SIFS}}+T_{\mathrm{C} T S}+T_{\mathrm{d} a t a}+T_{\mathrm{LIFS}}
\end{aligned}
$$

\subsection{LLDN-based TDMA}

Figure $2 \mathrm{~b}$ shows the other way to apply the LLDN to the cooperator-assisted WBAN. A superframe is divided into $2\left(N_{\text {node }}-1\right)$ timeslots, where the $(2 i-1)$ th and $2 i$ th timeslots are assigned by the BANCO to the $i$ th node and its cooperator, respectively. Whenever a cooperator successfully receives the packet of its parent node, it attempts to transmit its stored packet. Unlike the hybrid TDMA/CSMA, cooperative transmissions are always conducted whether the direct transmission fails or not.

In this case, the time slot duration $T_{\mathrm{t} s}$ needs to satisfy the following two criteria:

$$
\begin{aligned}
T_{\mathrm{S} F} & =M_{\mathrm{s}} / F \geq T_{\mathrm{ts}} \times\left(N_{\mathrm{node}}-1\right)+T_{\mathrm{beacon}}+T_{\mathrm{SIFS}} \\
T_{\mathrm{ts}} & \geq 2 \times\left(T_{\mathrm{d} a t a}+T_{\mathrm{LIFS}}\right)
\end{aligned}
$$

\section{Cooperator and BANCO selections}

We assume that the $k$ th node has been selected as a temporal BANCO.

\subsection{Optimal cooperator selection}

For every packet from the $i$ th source node, a node giving the highest packet success rate should be selected as its cooperator. Therefore, the optimal cooperator selection algorithm is summarized as Algorithm 1, where the metric in the maximization problem is the packet success rate for the two-hop link from the $i$ th source node to the BANCO via the $j$ th cooperator candidate, which is given by

$$
M_{i, j, k ; n}^{o p t}=P S R_{i, j, k}(n)=P S R_{i, j}(n) \times P S R_{j, k}(n) .
$$

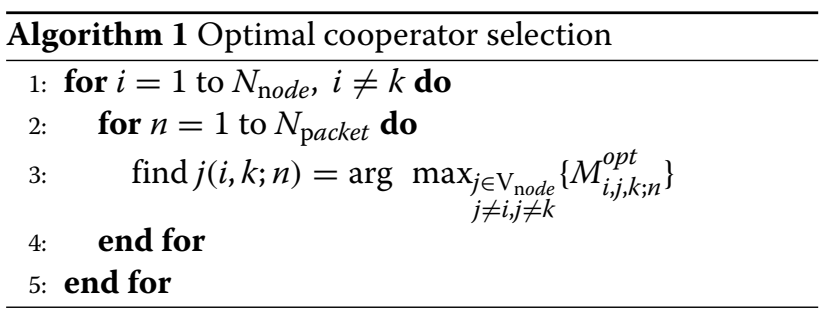

In Algorithm 1, for each source node, its cooperator can be selected independently packet by packet. The calculation of packet success rate may be the most computationally intensive, but focusing only on the cost of solving the maximization problem, the computational complexity of Algorithm 1 becomes $O\left(N_{\text {node }}^{2} N_{\text {packet }}\right)$ (see the Appendix 1). Note that the optimal cooperator selection is unrealistic in the sense that the BANCO needs to measure the packet success rates for the $i$ th source node via all the $j$ th nodes $\left(j \in \mathrm{V}_{\text {node }}, j \neq k, j \neq i\right)$ simultaneously packet by packet.

\subsection{Proposed cooperator selection}

In the proposed cooperator selection, the packet transmission is composed of two processes: a flooding process and a data transmission process. During the flooding process, the WBAN wearer takes different actions and postures, and the RSSIs of all links are measured at the same time by repeating a process where all nodes broadcast and re-broadcast their packets. After the flooding 


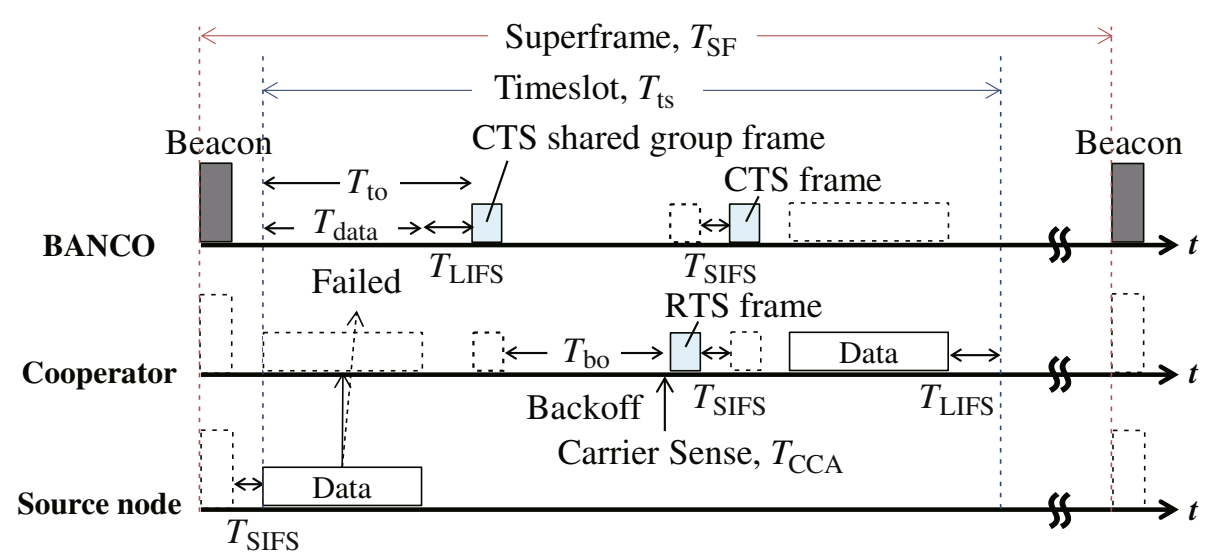

(a) LLDN with shared groups

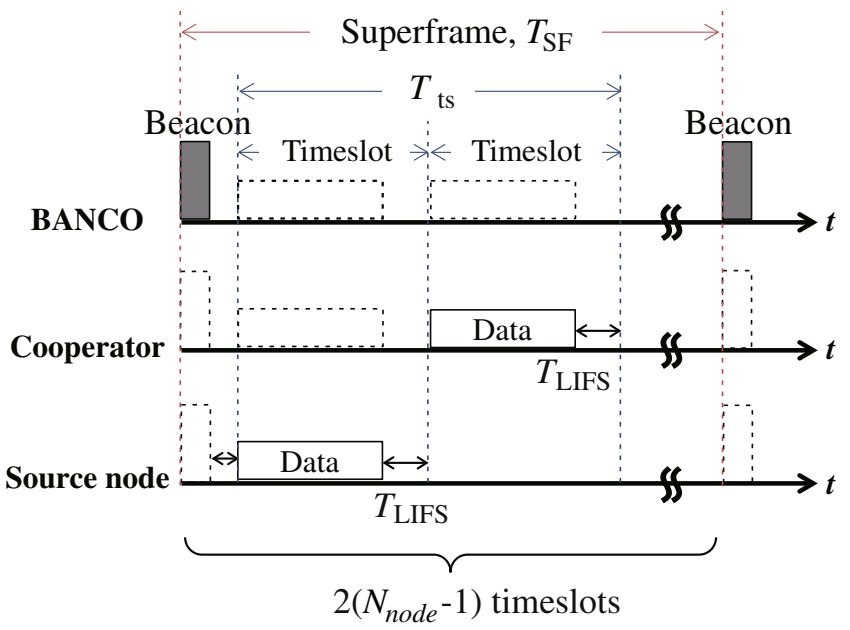

(b) LLDN without shared groups

Fig. 2 Superframe structures of the hybrid-TDMA/CSMA (a) and TDMA (b)

process, an adequate cooperator for each source node and a BANCO are selected based on the collected RSSIs information. Selected cooperators are considered to be moderately effective for the different actions and postures.

Hereby, we explain the flooding process in detail. Assume that each node broadcasts hello packets $N_{\text {hello }}$ times $\left(n=1, \cdots, N_{\text {hello }}\right)$. When a node directly receives a hello packet from another node, the node measures the RSSI of the packet and re-broadcasts only once the packet adding the measured RSSI in it. When a node receives a rebroadcast hello packet, the node no longer re-broadcasts it. In this case, defining two sets of the indices of the hello packets which are transmitted from the $i$ th source node and received at the BANCO directly and via the jth cooperator candidate as $Z_{i k}^{1}$ and $Z_{i j k}^{2}$ with their elements $n_{i k}^{1}$ $\left(n_{i k}^{1} \in \mathrm{Z}_{i k}^{1}\right)$ and $n_{i j k}^{2}\left(n_{i j k}^{2} \in \mathrm{Z}_{i j k}^{2}\right)$, respectively, the $k$ th node can know the RSSI of the first link as $R S S I_{i j}\left(n_{i j k}^{2}\right)$ and that of the second link as $\operatorname{RSSI}_{j k}\left(n_{i j k}^{2}\right)$.
Practically, a node should not cooperate too many source nodes, because it shortens the lifetime of WBAN. For instance, when a node acts as cooperators for three source nodes, the lifetime of the WBAN becomes $25 \%$ of the lifetime of a WBAN without cooperator assistance. Therefore, the number of nodes which a node can cooperate should be limited, and hereafter, we define $\mu(j)$ and $N_{\text {cmax }}$ as the multiplicity of the $j$ th cooperator candidate and its allowable largest value, respectively. The proposed strict cooperator selection algorithm is summarized as Algorithm 2, where the metric in the maximization problem is modified as

$$
M_{i, j, k}^{s t r}=\sum_{n_{i j k}^{2} \in Z_{i j k}^{2}} P S R_{i j k}\left(n_{i j k}^{2}\right) .
$$

Algorithm 2 is mathematically not tractable, since there are too many candidates to be evaluated for finding the optimal solution for each $j$ and furthermore 


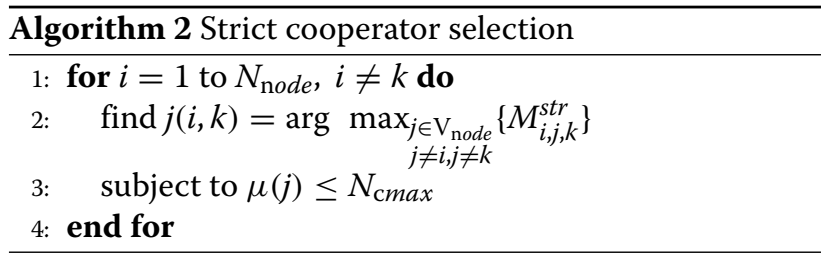

selectable cooperator candidates for one source node is affected by those for other source nodes. In fact, the computational complexity of the algorithm is lowerbounded as $O\left(\left(N_{\text {node }}-1\right)\right.$ !) (see the Appendix 2$)$. Therefore, we need to simplify Algorithm 2.

\subsubsection{Without multiplicity constraint}

For the case of $N_{\text {cmax }}=N_{\text {node }}-2$, we can select a cooperator independently for each source node, in other words, we can simply remove the constraint from Algorithm 2. Here, we introduce one more simplifications, that is, replacement of the packet success rate calculation by an RSSI calculation, taking into consideration the fact that the packet success rate is a monotonous increasing function on the RSSI and the packet success rate through a two-hop link is more affected by the one in a worse link:

$$
\begin{aligned}
M_{i, j, k}^{s m p} & =\sum_{n_{i j k}^{2} \in \mathrm{Z}_{i j k}^{2}} \operatorname{RSSI}_{i j k}\left(n_{i j k}^{2}\right) \\
\operatorname{RSSI}_{i j k}\left(n_{i j k}^{2}\right) & =\min \left\{R S S I_{i j}\left(n_{i j k}^{2}\right), \operatorname{RSSI}_{j k}\left(n_{i j k}^{2}\right)\right\} .
\end{aligned}
$$

In this case, the simplified cooperator selection algorithm without multiplicity constraint is summarized as Algorithm 3. Its computational complexity is $O\left(N_{\text {node }}^{2}\right)$ (see the Appendix 3).

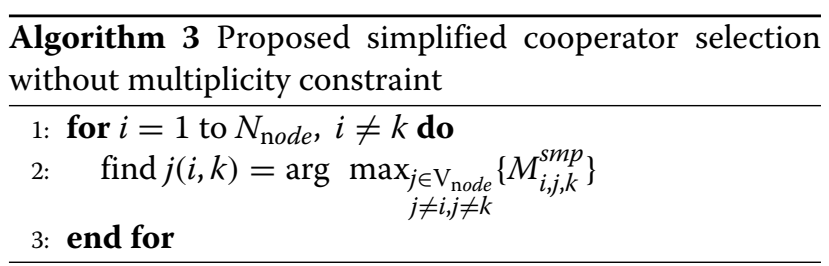

\subsubsection{With multiplicity constraint}

Now, in terms of source nodes, let us define a set of the indices of cooperator candidates for the $i$ th source node as

$$
\begin{array}{r}
C_{i k}=\left\{c_{i k q} ; c_{i k q}=j(i, k)=\arg \underset{\substack{q \operatorname{thmax} \max \\
j \in \mathrm{V}_{\text {node }}}}{j \neq i, j \neq k}\left\{M_{i, j, k}^{s m p}\right\},\right. \\
\left.q=1,2, \cdots, N_{\text {node }}-2\right\}
\end{array}
$$

where $q \mathrm{t} h \max \{(\cdot)\}$ denotes the function picking up the $q$ th maximum out of $(\cdot)$. In (15), $c_{i k q}$ is the $q$ th element of $\mathrm{C}_{i k}$ which has the essentiality metric as

$$
\varepsilon_{i k q}=\underset{\substack{j \in \mathrm{V}_{\text {node }} \\ j \neq i, j \neq k}}{q \mathrm{th} \max }\left\{M_{i, j, k}^{s m p}\right\}-\left(q+\underset{\substack{j \in \mathrm{V}_{\text {node }} \\ j \neq i, j \neq k}}{1) \operatorname{th} \max }\left\{M_{i, j, k}^{s m p}\right\}\right.
$$

and the cooperator candidate $c_{i k q}$ with larger $\varepsilon_{i k q}$ is more essential for the $i$ th source node. In addition, let us define the number of packets lost in the direct link from the $i$ th source node to the BANCO as the unreliability metric:

$$
v_{i k}=N_{\text {hello }}-\mathrm{Z}_{i k}^{1}
$$

where the direct link of the $i$ th source node with larger $v_{i k}$ is less reliable.

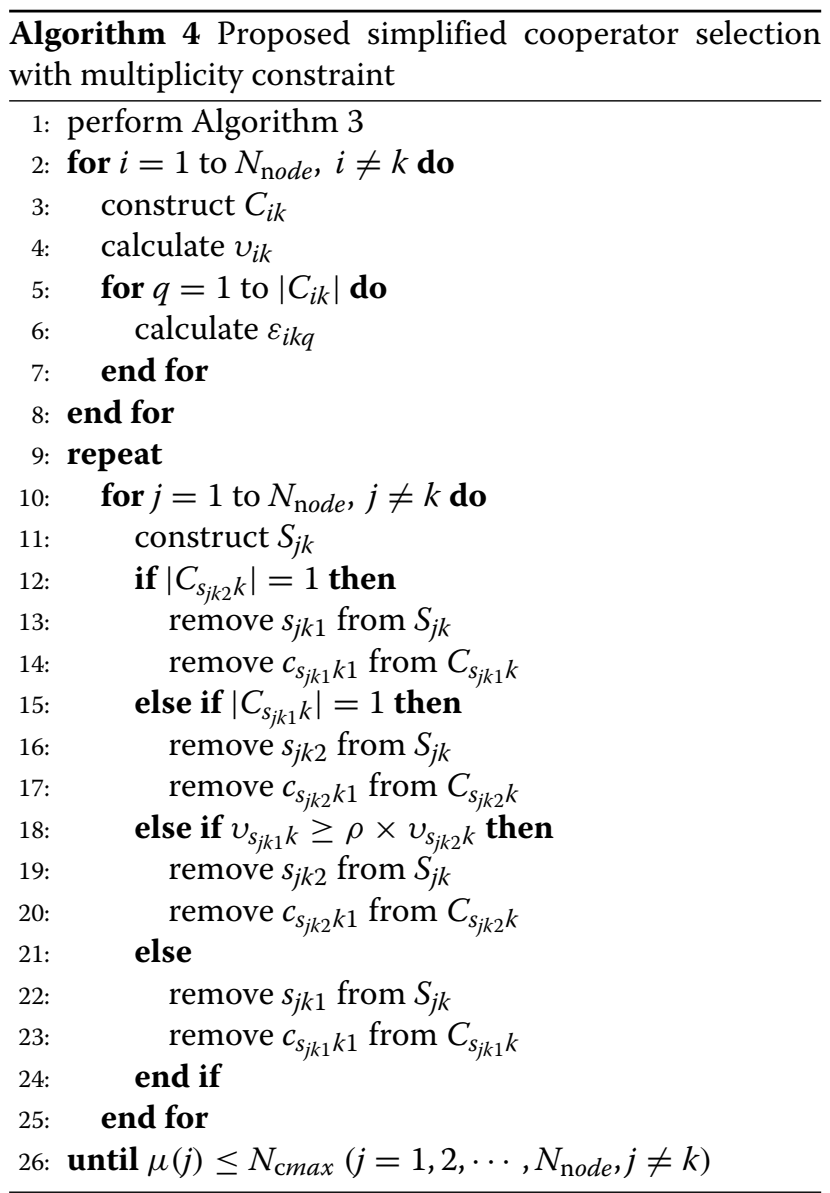

On the other hand, in terms of cooperator candidates, let us define a set of the indices of (parent) source nodes for the $j$ th cooperator candidate as

$$
\mathrm{S}_{j k}=\left\{s_{j k u} ; s_{j k u}=i(j, k)=\arg \underset{\substack{u \mathrm{t} h \min \\ i \neq j, i \neq k, \mathrm{~V}_{\text {nod }} \\ i \neq j}}{\left.i \neq \varepsilon_{i k 1}\right\}}\right\}
$$


where $u$ th $\min \{(\cdot)\}$ denotes the function picking up the $u$ th minimum out of (.), and the source node $s_{j k u}$ with smaller $u$ is more removable from $S_{j k}$.

In this algorithm, we first apply the cooperator selection without multiplicity constraint given by Algorithm 3 and then we remove parent source nodes for cooperator candidates whose multiplicities are more than the allowable largest value. Let us assume that the $j$ 'the cooperator candidate has $N_{\mathrm{c} \text { max }}^{\prime}>N_{\mathrm{c} \max }$ parent source nodes:

$$
\mathrm{S}_{j^{\prime} k}=\left\{s_{j^{\prime} k 1}, s_{j^{\prime} k 2}, \cdots, s_{j^{\prime} k u}, \cdots, s_{j^{\prime} k N_{\text {cmax }}^{\prime}}\right\} .
$$

The element $s_{j^{\prime} k u}$ with smaller $u$ has the smaller essentiality metric on $j^{\prime}$ the cooperator candidate, so in this sense, it is quite natural to examine the removability from $s_{j^{\prime} k 1}$. However, when comparing the unreliability metrics of $s_{j^{\prime} k 1}$ and $s_{j^{\prime} k 2}$, namely, $v_{s_{j^{\prime}} k_{1} k}$ and $v_{s_{j^{\prime}} k 2}$, if $v_{s_{j^{\prime} k 1} k}>$ $v_{s^{\prime} k 2 k}$, then it may be better not to remove $s_{j^{\prime} k 1}$, because it implies that $s_{j^{\prime} k 1}$ requires more help from a cooperator. Therefore, in the proposed algorithm, every examination the elements of $S_{j k}$, either of $s_{j k 1}$ or $s_{j k 2}$ is always removed from $S_{j k}$, which satisfies a certain condition. The simplified cooperator selection algorithm with multiplicity constraint is summarized as Algorithm 4, where the "remove" operation contains renumbering the remaining elements after the removal, and $\rho$ is a scaling factor for the unreliability comparison. Its computational complexity is $O\left(N_{\text {node }}^{3}\right)$ (see the Appendix 4).

\subsubsection{Proposed BANCO selection}

After an adequate cooperator has been selected for each source node when temporally selecting the $k$ th node as a BANCO $\left(k=1,2, \cdots, N_{\text {node }}\right)$, the BANCO is finally selected. Here, it is reasonable that the adequate BANCO should receive as many packets with larger RSSIs as possible from all other nodes directly or indirectly. Therefore, based on the minmax criterion which can ensure that all nodes' packet error rates are not extremely high, the metric of the BANCO selection can be given by

$$
\begin{aligned}
M_{k}^{B A N C O} & =\min _{\substack{i \in V_{\text {node }} \\
i \neq j}}\left\{M_{k, i}^{B A N C O}\right\} \\
M_{k, i}^{B A N C O} & =\sum_{n_{i k}^{1} \in Z_{i k}^{1}} \operatorname{RSSI}_{i k}\left(n_{i k}^{1}\right) \\
& +\sum_{n_{i j(i, k) k}^{2} \in Z_{i j(i, k) k}^{2}} \operatorname{RSSI}_{i j(i, k) k}\left(n_{i j(i, k) k}^{2}\right) .
\end{aligned}
$$

The BANCO selection algorithm is summarized as Algorithm 5. For each temporally selected BANCO $(k=$ $\left.1,2, \cdots, N_{\text {node }}\right)$, the BANCO selection metric is calculated, so its computational complexity is $O\left(N_{\text {node }}^{2}\right)$ (see the Appendix 5).

\begin{tabular}{l} 
Algorithm 5 Proposed BANCO selection \\
1: find $k=\arg \max _{k \in N_{\text {node }}}\left\{M_{k}^{B A N C O}\right\}$ \\
\hline
\end{tabular}

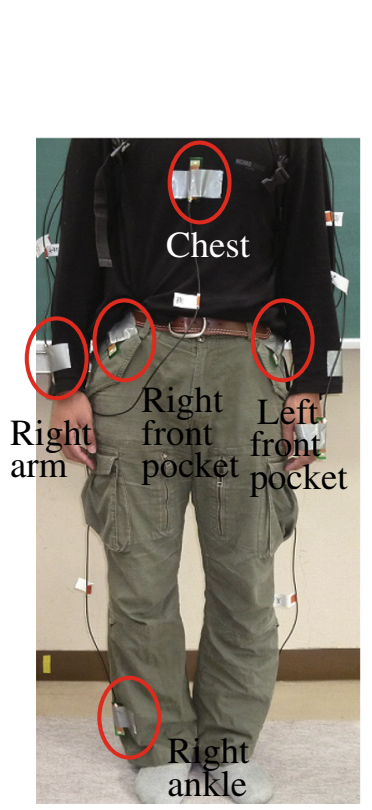

(a) Front view

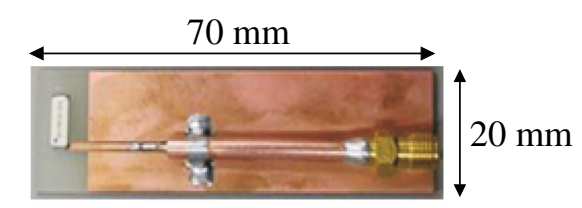

(d) Chip antenna

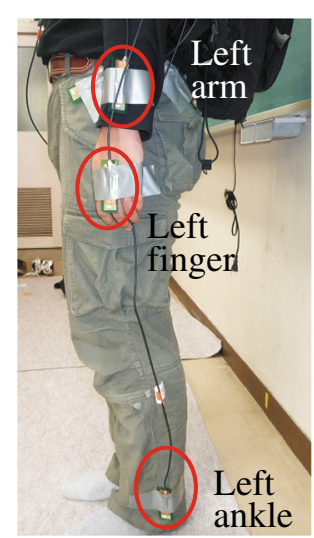

(b) Side view

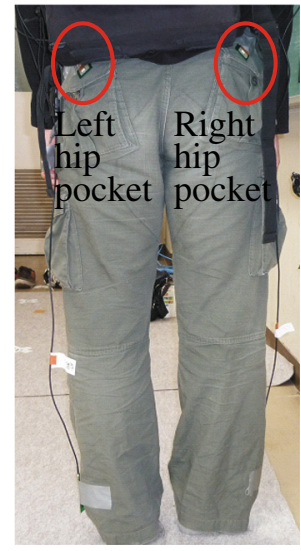

(c) Rear view

Fig. 3 Positions of ten chip antennas: front view $(\mathbf{a})$, side view $(\mathbf{b})$, rear view $(\mathbf{c})$, and chip antenna $(\mathbf{d})$ 
Table 1 Specifications of the chip antenna

\begin{tabular}{ll}
\hline Description & Value \\
\hline Length & $8 \mathrm{~mm}$ \\
Width & $3 \mathrm{~mm}$ \\
Height & $1 \mathrm{~mm}$ \\
Gain & $2 \mathrm{dBi} \max$ \\
e VSWR & $2 \mathrm{max}$ \\
\hline
\end{tabular}

\section{Experiment on RSSI measurement}

We conducted an experiment for obtaining RSSI data in our university campus, where we used a wireless signal based on the IEEE 802.15.4 standard with bandwidth of $2 \mathrm{MHz}$ and transmission power of $0 \mathrm{dBm}$ in the 2.4 GHz-ISM band. As shown in Fig. 3, we attached ten chip antennas to a subject, where their locations were as follows; right arm, left arm, right ankle, left ankle, chest, left finger, right front pocket, left front pocket, right hip pocket, and left hip pocket. Table 1 and Fig. 4 show the specification and radiation pattern of the chip antenna, respectively.

In each session of the experiment, a packet was broadcast from each antenna every $0.1 \mathrm{~s}$ for $150 \mathrm{~s}$ in order to measure RSSIs at its other nine antennas. Figure 5 shows the route in one session of the experiment, and as shown in the figure, we conducted the experiment in a mixed indoor/outdoor environment. The subject took different postures and actions, such as sitting (20 s), standing up, standing still (20 s), turning round (20 s) in a room, walking (30 s in a corridor and $40 \mathrm{~s}$ outdoors), sitting down, and sitting still (20 s) outdoors. The same experiment was performed on three subjects.

Figure 6 shows the temporal RSSI variation for the rightankle to left-finger link for subject A. As can be seen in the figure, the RSSI dynamically changes depending on the postures and actions. The other links had similar tendencies to this link. From Fig. 4, the radiation pattern of the chip antenna has no deep notch in both the horizontal and vertical planes, so we can see that the RSSI variation in Fig. 6 is mainly due to blocking of the link by parts of the body of subject $A$.

\section{Performance evaluation and discussions}

First of all, Table 2 summarizes the system parameters required for MAC design and performance evaluation of the proposed WBAN.

\subsection{Superframe design}

We designed superframe parameters satisfying the criteria given by (7) and (8) for the hybrid-TDMA/CAMA whereas given by (9) and (10) for the TDMA. Table 3 summarizes the designed superframe parameters. In summary, according to the IEEE 802.15.4e LLDN, the hybrid-TDMA/CSMA and TDMA can accommodate ten nodes in a BAN each of which sense vital signs 100 times per second and transmits the data 10 times per second.

\subsection{Computer simulation and results on the packet error rate}

We conducted computer simulations to evaluate the packet error rate of the cooperator-assisted WBAN with the designed superframe parameters using the stored RSSI data obtained in the experiment. One simulation session is repeated until each node broadcasts 3000 hello packets in the flooding process and transmits 10,000 data packets in the data transmission process for each case of all BANCO's positions. We confirmed that there was little difference

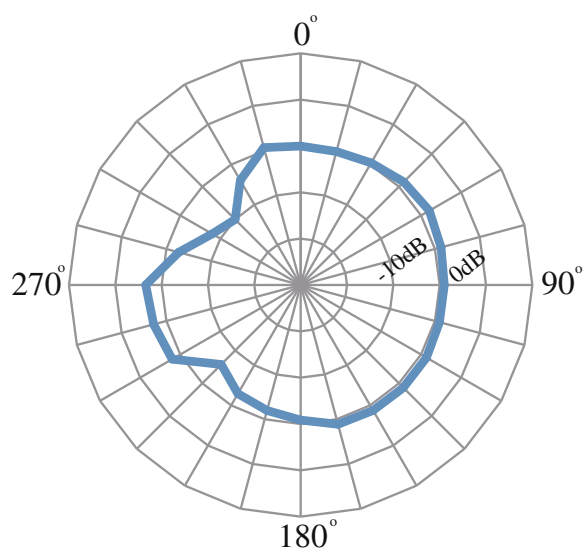

(a) Horizontal plane

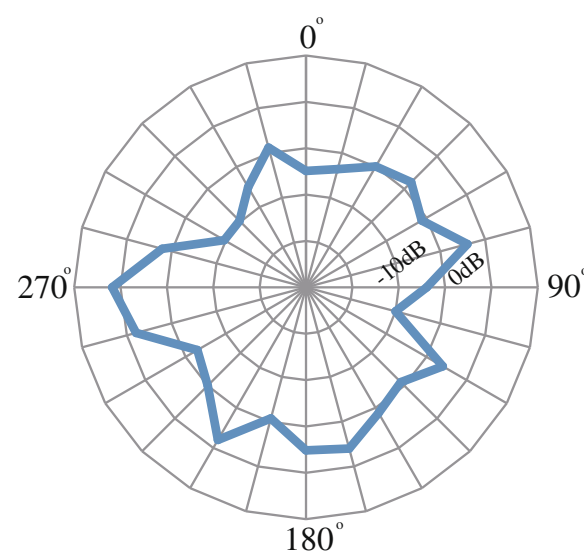

(b) Vertical plane

Fig. 4 Radiation pattern of the chip antenna: horizontal plane (a) and vertical plane (b) 


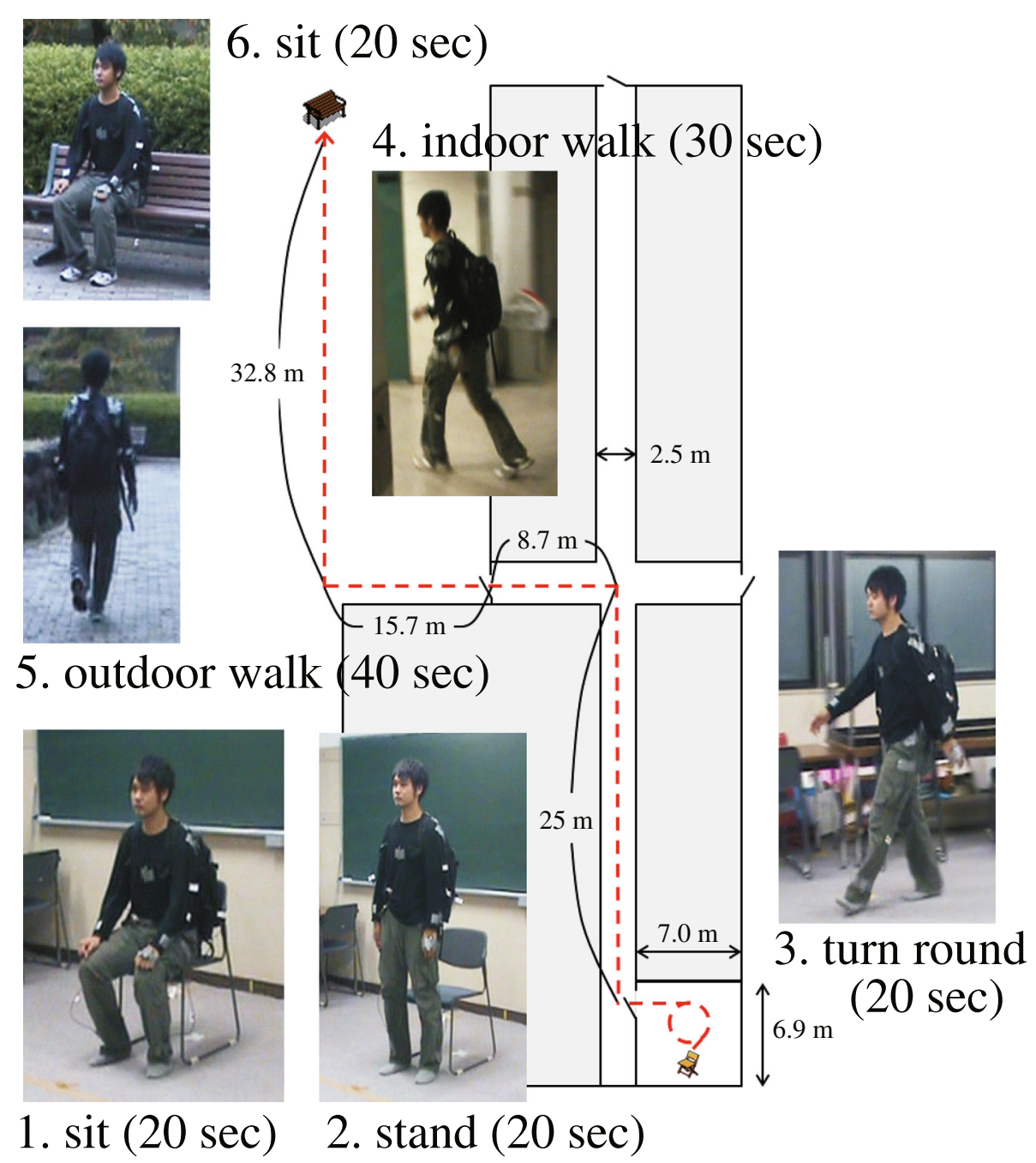

Fig. 5 Route of the experiment

in the packet error rate between the hybrid-TDMA/ CSMA and TDMA, so in the following figures, the packet error rate is common to the two MACs.

Figure 7 shows the average packet error rate for the cooperator-assisted BAN without cooperator multiplicity constraint. For comparison purpose, besides the optimal and proposed cooperator selections, this figure contains the performance for a single-hop (without packet retransmission), a self-retransmission (without cooperator assistance), and a random cooperator selection (a cooperator is randomly selected for each node, and it is changed periodically every $30 \mathrm{~s}$ ). Each result is averaged over the three subjects. It can be seen from the figure that the self-retransmission gets little improvement. The most obvious reason is that the link quality cannot be improved in such a short duration. It can be also seen that the random cooperator selection does not greatly improve the performance. It is because, when a cooperator happens to be selected which gives a higher blocking correlation between the direct and indirect paths between its parent node and the BANCO, the cooperator assistance does not work well at all. When the transmission power is $-10 \mathrm{dBm}$ which is considered to be a typical transmission power of a WBAN, the average packet error rate of the single-hop transmission scheme without re-transmission is about $5.22 \%$, on the other hand, that of the proposed cooperator/BANCO selection is about $0.103 \%$, and there is no packet loss with the optimal cooperator selection. Considering the optimal cooperator selection is unrealizably changing the best cooperator packet by packet whereas the proposed cooperator selection is practical keeping the same cooperator for the whole data transmission process, we believe that the proposed cooperator selection significantly improves the performance. 


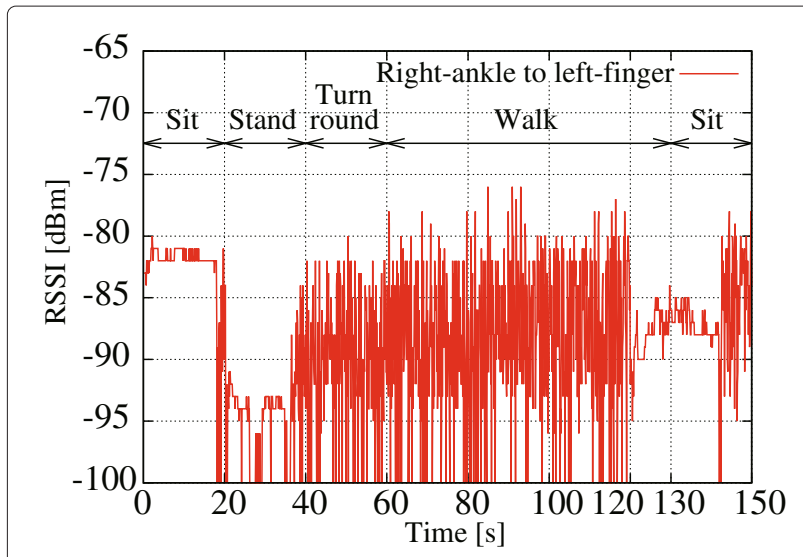

Fig. 6 RSSI variation for the right-ankle to left-finger link (subject A)

Figures $8 \mathrm{a}-\mathrm{c}$ show the packet error rates against the position of the BANCO for subjects $\mathrm{A}, \mathrm{B}$, and $\mathrm{C}$, respectively. Here, the transmission power is $-10 \mathrm{dBm}$, and no cooperator multiplicity constraint is imposed. In each of the figures, the position selected by the proposed BANCO selection is also shown. It can be seen from the figures that the packet error rate largely depends on the position of the BANCO; if an inappropriate BANCO position is selected, the obtained packet error rate is terribly high, and furthermore, the optimal BANCO position changes when the subject changes. The proposed cooperator/BANCO selection can reduce the packet error rate of the single-hop transmission by around $1 / 250$. However, as a cooperator for each source node, it can almost always select the node giving lower packet error rate but cannot always select the node giving the lowest packet error rate. To select a BANCO, the proposed algorithm uses the sum of RSSIs through the direct and indirect links to each source node, in other words, only the average (first moment) of the RSSI temporal variation (see (21)). In reality, the packet error rate depends on not simply the average but complicatedly the distribution of the RSSI, so to improve the BANCO

Table 2 System parameters

\begin{tabular}{lll}
\hline Description & Parameter & Value \\
\hline Data transmission rate & $R$ & $250 \mathrm{kbits} / \mathrm{s}$ \\
Number of nodes & $N_{\text {node }}$ & 10 nodes \\
Sampling rate & $F$ & $100 \mathrm{~Hz}$ \\
Number of sensors & $N_{\text {sensor }}$ & 3 sensors/node \\
A/D resolution & $q$ & 8 bits \\
Additional information & $d$ & 16 bits \\
Packet overhead length & $L_{0}$ & 72 bits \\
Number of aggregated date per packet & $M_{\mathrm{s}}$ & 10 data \\
Noise figure & $N F$ & $10 \mathrm{~dB}$ \\
Thermal noise density & $N_{\text {noise }}$ & $-174 \mathrm{dBm} / \mathrm{Hz}$ \\
Channel bandwidth & $B_{\text {ch }}$ & $2 \mathrm{MHz}$ \\
\hline
\end{tabular}

Table 3 Designed superframe parameters

\begin{tabular}{lll}
\hline Description & Parameter & Value \\
\hline Data packet duration & $T_{\text {data }}$ & $1.89 \mathrm{~ms}$ \\
Clear channel assessment & $T_{\text {CCA }}$ & $0.128 \mathrm{~ms}$ \\
Beacon & $T_{\text {beacon }}$ & $0.416 \mathrm{~ms}$ \\
CTS shared group frame & $T_{\text {CTSSG }}$ & $0.384 \mathrm{~ms}$ \\
RTS frame & $T_{\text {RTS }}$ & $0.416 \mathrm{~ms}$ \\
CTS frame & $T_{\text {CTS }}$ & $0.416 \mathrm{~ms}$ \\
Long interframe space & $T_{\text {LIFS }}$ (TDMA/CSMA) & $0.64 \mathrm{~ms}$ \\
& $T_{\text {LIFS }}$ (TDMA) & $3.16 \mathrm{~ms}^{1}$ \\
Short interframe space & $T_{\text {SIFS }}$ & $0.192 \mathrm{~ms}$ \\
Timeout time & $T_{\text {to }}$ & $4.5 \mathrm{~ms}^{\circ}$ \\
Backoff time & $T_{\text {bo }}$ & $2.24 \mathrm{~ms}^{2}$ \\
Timeslot duration & $T_{\text {ts }}$ & $11.0 \mathrm{~ms}^{2}$ \\
\hline
\end{tabular}

${ }^{1}$ We set the value of TLFS (TDMA) so that the hybrid-TDMA/CSMA and TDMA have the same duration of a superframe

${ }^{2}$ For the sake of simplicity, in the TDMA, $T_{\text {ts }}$ is assumed to be the duration of two slots

selection performance, we need to take into consideration its higher-order moments in the algorithm. On the other hand, in some applications, it is required to select the BANCO position in advance. The results indicate that "left waist" is an adequate position as the BANCO for all subjects.

Figure 9 shows the average packet error rate for the duration of RSSI data used in the flooding process. It is clear that, even though the flooding duration is short, if the WBAN wearer can take different actions and postures in it, the proposed cooperator/BANCO selection improves the packet error rate.

Figure 10 shows the dependency of the packet error rate on the scaling factor $(\rho)$ for the unreliability comparison in the cooperator selection with multiplicity constraint (see line 18 in Algorithm 4). From the result, we set $\rho=1.5$

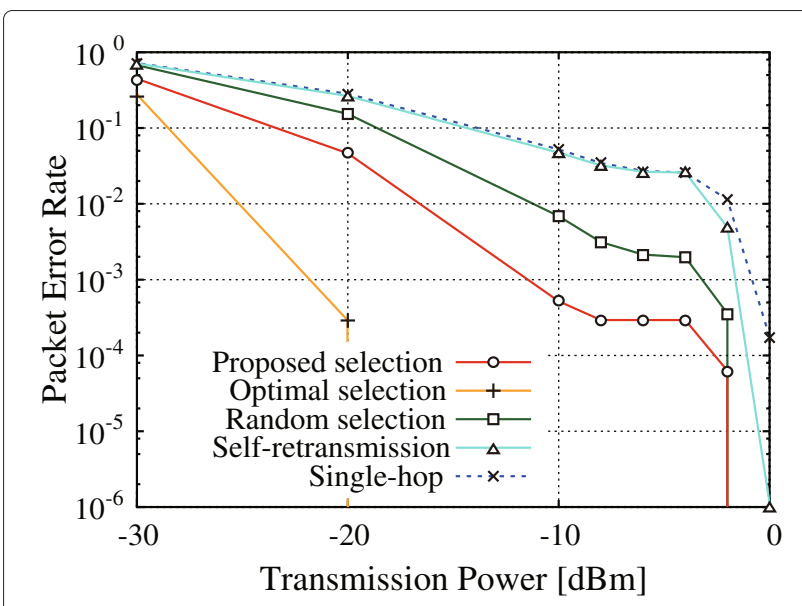

Fig. 7 Average packet error rate without cooperator multiplicity constraint 


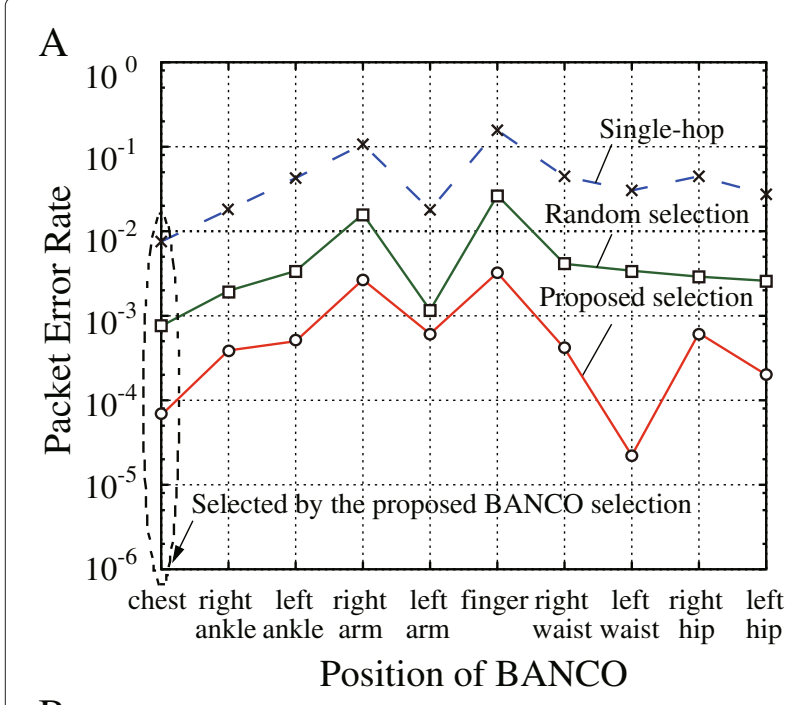

B

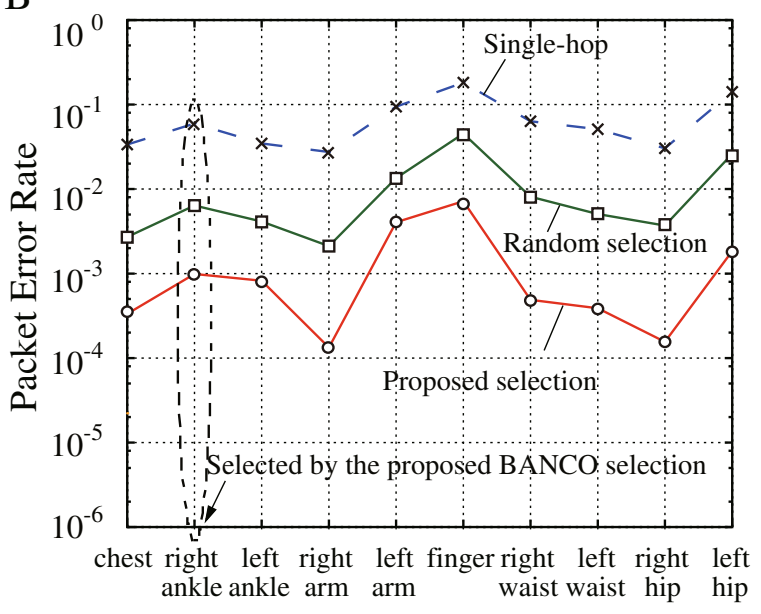

Position of BANCO

$\mathrm{C}$

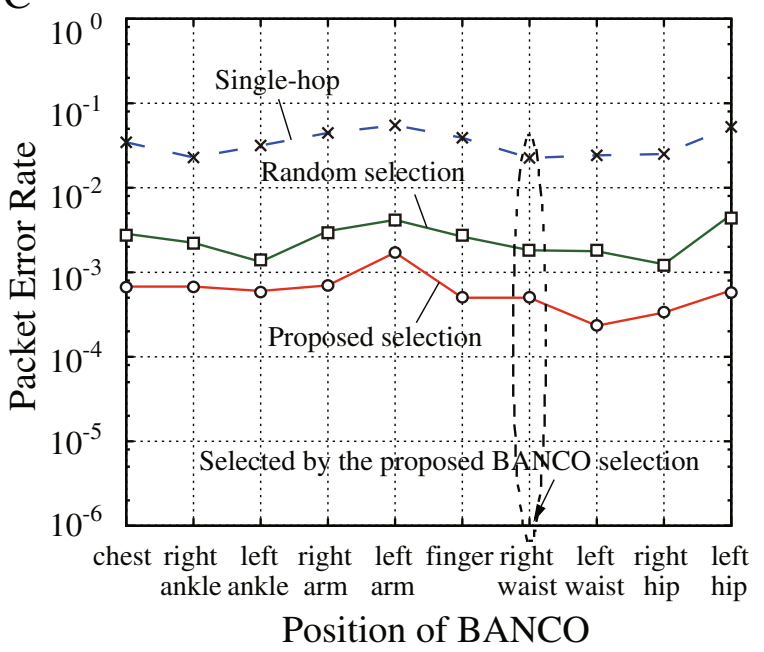

Fig. 8 Packet error rate without cooperator multiplicity constraint (transmission power $=-10 \mathrm{dBm}$ ), a: subject $\mathrm{A}$, b: subject $\mathrm{B}$ and c: subject C

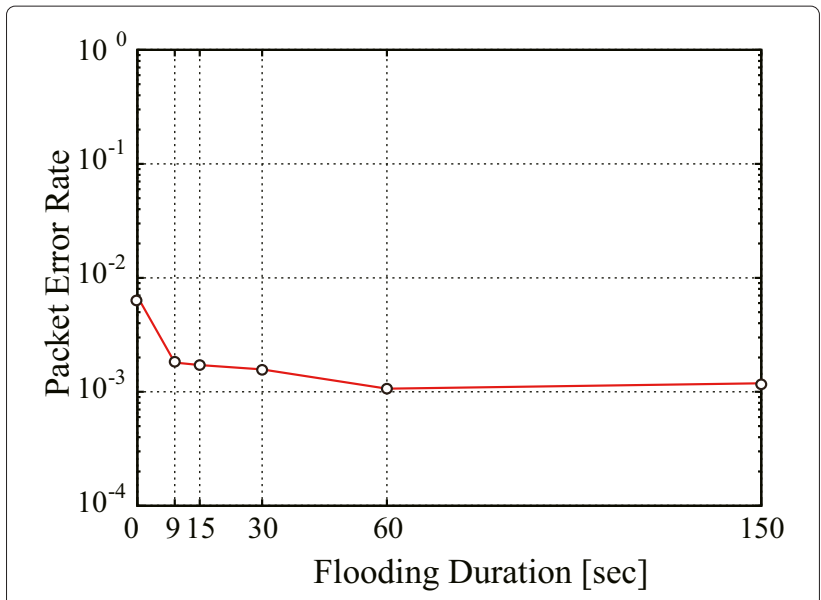

Fig. 9 Flooding duration versus the packet error rate without cooperator multiplicity constraint (transmission power $=-10 \mathrm{dBm}$ )

in the following, but there is no large dependency on $\rho$ observed in the figure, so we may simply compare $v_{s_{j k 1} k}$ with $v_{s_{j k 2} k}$ just setting $\rho=1.0$.

Figure 11 shows the results where $N_{\text {cmax }}=1,2,4$, and $\rho=1.5$ for the cooperator selection with multiplicity constraint. The packet error rate with $N_{\mathrm{cmax}}=1$ is likely to be worse than the packet error rates with $N_{\mathrm{c} \max }=2$ and 4 for almost all BANCO positions, but between with $N_{\text {cmax }}=2,4$, and without multiplicity constraint, there is no large difference in the packet error rates. This implies that, in the WBAN with ten nodes on different positions of a human body, any node has multiple better cooperator candidates. From the result, we can see that $N_{\text {cmax }}=2$ must be fair in terms of both the packet error rate and energy saving for cooperators.

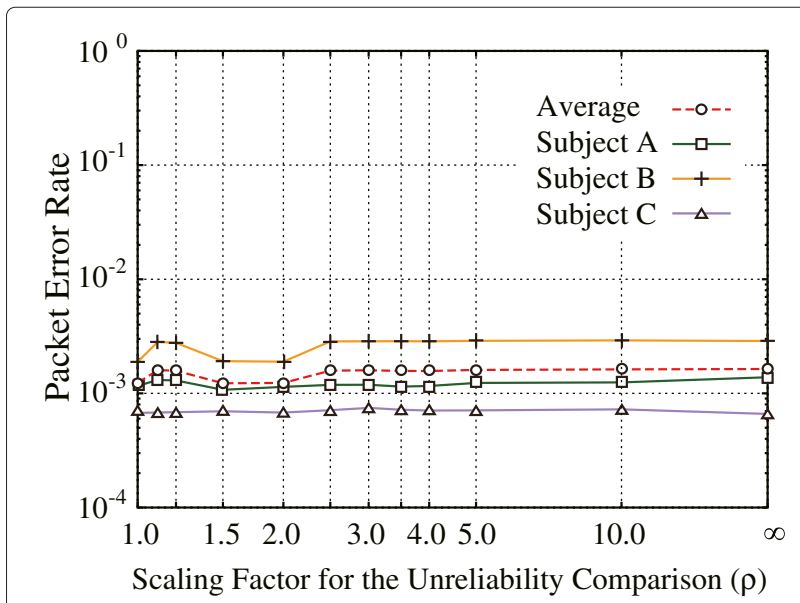

Fig. 10 Scaling factor for the unreliability comparison versus the packet error rate with cooperator multiplicity constraint $(-10 \mathrm{dBm}$, $N_{\text {cmax }}=2$ ) 


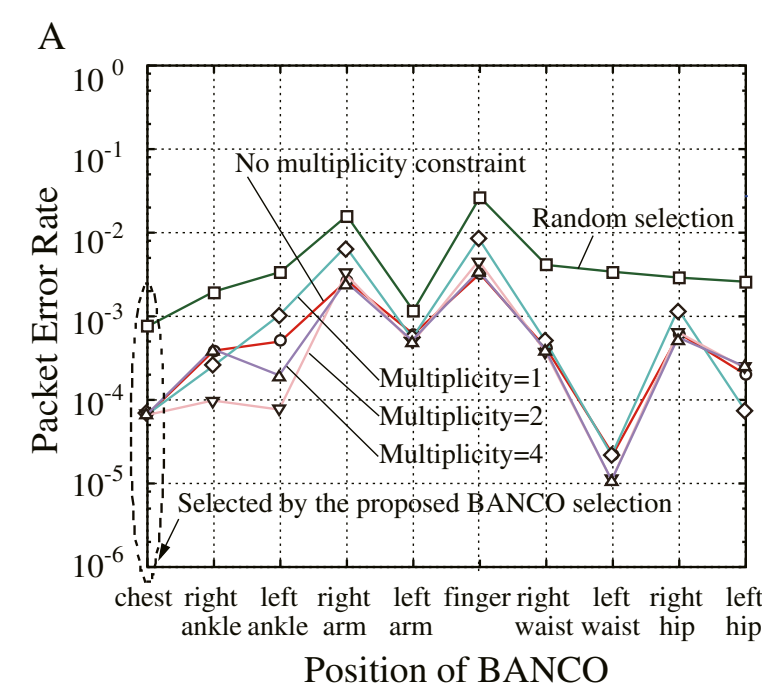

B

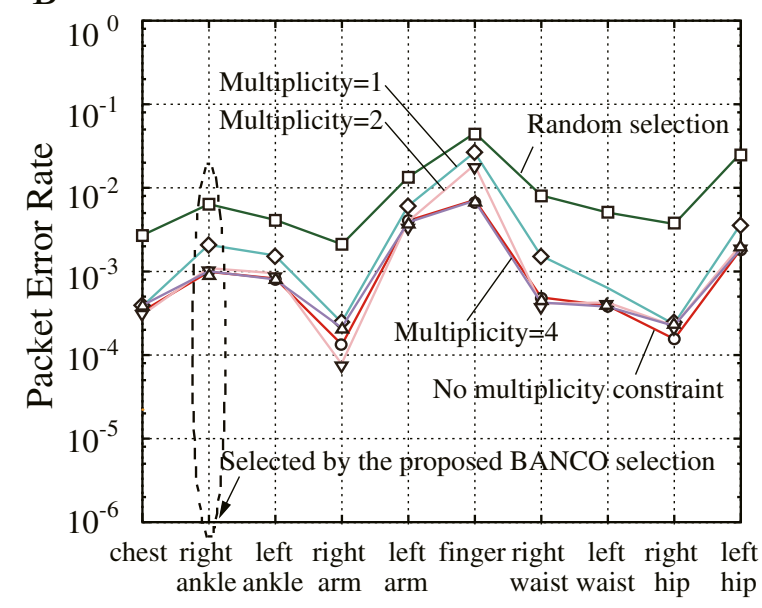

Position of BANCO

$\mathrm{C}$

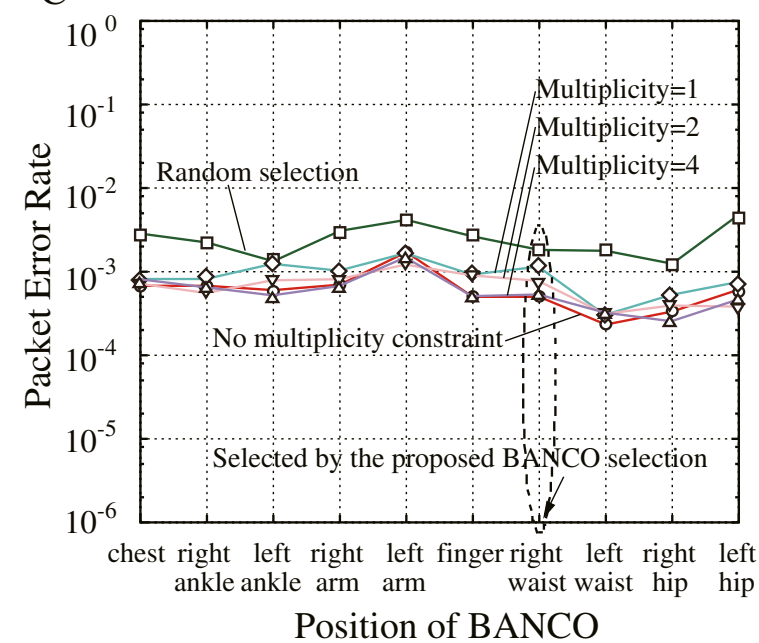

Fig. 11 Packet error rate with cooperator multiplicity constraint (transmission power $=-10 \mathrm{dBm}$ ), $\mathbf{a}$ : subject $\mathrm{A}$, $\mathbf{b}$ : subject $\mathrm{B}$ and $\mathbf{c}$ : subject C
Table 4 Consumption current values for a typical RF module

\begin{tabular}{ll}
\hline State & Consumption current \\
\hline Transmission & $24 \mathrm{~mA}$ \\
Reception & $20 \mathrm{~mA}$ \\
Standby & $1.3 \mu \mathrm{A}$ \\
\hline
\end{tabular}

Finally, we compare the power consumption between the hybrid-TDMA/CDMA and TDMA. Table 4 summarizes the consumption current values for a typical transceiver module [24], and Table 5 shows the result, where the power consumption of the TDMA/CSMA is normalized by that of the TDMA. Since the current consumption for reception is as high as that for transmission, the power consumption for the TDMA/CSMA is not much lower than that for the TDMA although cooperators transmit their stored packets only when the packet transmissions by their parents' nodes fail in the TDMA/CSMA.

\section{Conclusion}

In this paper, we have shown that, to enable a cooperatorassisted WBAN, a hybrid-TDMA/CSMA is realizable by making effective use of CTS shared group frame, and a TDMA is also realizable in the IEEE 802.15.4e LLDN. In addition, we have evaluated the packet error rate of a cooperator/BANCO selection based on both the hybridTDMA/CSMA and TDMA by computer simulations, where we have used the stored RSSI data obtained from a mixed indoor/outdoor environment with three subjects.

The proposed cooperator/BANCO selection works well in the realistic environment with typical transmission power of $-10 \mathrm{dBm}$, and the proposed cooperator selection is superior to the random selection by about $8 \mathrm{~dB}$. It is also shown by our computer simulations that it is important to select adequate cooperators for each subject. The TDMA was slightly disadvantageous over hybridTDMA/CSMA in terms of power consumption. However, the TDMA is simple; it requires no CCA, CTS frame and RTS frame, so it can shorten the timeslot duration thus accommodate more nodes for the same data packet duration. This must be the advantage of the TDMA.

The proposed BANCO selection was not able to always select the best node as the BANCO for the three subjects. To investigate a more efficient BANCO selection using higher-order moments of RSSI variation at the sacrifice of computational complexity must be one of our future works.

Table 5 Power consumption comparison

\begin{tabular}{ll}
\hline MAC & Power consumption \\
\hline TDMA & 1.0 \\
TDMA/CSMA & 0.76 \\
\hline
\end{tabular}




\section{Appendix 1}

For each $i\left(i=1,2, \cdots, N_{\text {node }}, i \neq k\right)$, and each $n(n=1$, $\left.2, \cdots, N_{\text {packet }}\right)$, we need to find the maximum out of a set of $M_{i, j, k ; n}^{o p t}$ with the number of elements $=N_{\text {node }}-2$. Since finding the maximum out of a set of $N$ values requires $N$ comparisons, the computational complexity of Algorithm is given by

$$
\begin{aligned}
C C_{1}=O\left(\left(N_{\text {node }}-2\right)\right. & \times\left(N_{\text {node }}-1\right) \\
& \left.\times N_{\text {packet }}\right) \propto O\left(N_{\text {node }}^{2} N_{\text {packet }}\right) .
\end{aligned}
$$

\section{Appendix 2}

When $N_{\text {cmax }}=1$, the computational complexity of Algorithm 2 is minimized, since the number of combinations of cooperator candidates is minimized. Although each source node cannot select itself as its cooperator, but its number is approximated as $\left(N_{\text {node }}-2\right)$ !. Therefore, the computational complexity of Algorithm is lower-bounded as

$$
C C_{2}=O\left(\left(N_{\text {node }}-2\right) ! \times\left(N_{\text {node }}-1\right)\right)=O\left(\left(N_{\text {node }}-1\right) !\right) .
$$

\section{Appendix 3}

For each $i\left(i=1,2, \cdots, N_{\text {node }}, i \neq k\right)$, we need to find the minimum out of a set of $M_{i, j, k}^{s m p}$ with the number of elements $=N_{\text {node }}-2$, so the computational complexity of Algorithm 3 is given by

$$
C C_{3}=O\left(N_{\text {node }}^{2}\right) \text {. }
$$

\section{Appendix 4}

In Algorithm 4, the computation complexities of performing Algorithm 3 (line 1) is given by

$$
C C_{4}^{\text {algo3 }}=O\left(N_{\text {node }}^{2}\right) \text {. }
$$

In addition, taking into consideration that the computational complexity of sorting a set of $N$ values is given by $O\left(N^{2}\right)$ for the worst case [25], the computational complexities of constructing $C_{i k}$ for $i=1,2, \cdots, N_{\text {node }}, i \neq k$ (line 2-8) and $S_{j k}$ for $j=1,2, \cdots, N_{\text {node }}, j \neq k$ (line 9-26) are respectively given by

$$
\begin{aligned}
& C C_{4}^{C}=O\left(\left(N_{\text {node }}-2\right)^{2} \times\left(N_{\text {node }}-1\right)\right) \propto O\left(\left(N_{\text {node }}^{3}\right)\right. \\
& C C_{4}^{S} \propto O\left(\left(N_{\text {node }}^{3}\right) .\right.
\end{aligned}
$$

The repeat-until loops furthermore contains comparison of unreliability metrics (line 18), so we need to count the number of the comparisons. Let us consider the worst case where the number of comparisons becomes the largest. In the first round of parent node removal, it happens when the best cooperator candidates of all source nodes $\left(N_{\text {node }}-2\right)$ are the same. In this case, the cooperator candidate needs to compare the unreliability metrics of its parent source nodes $N_{\text {node }}-2-N_{\text {cmax }}$ times. Then, in the second round of parent node removal, it happens when the best cooperator candidates of remaining source nodes $\left(N_{\text {node }}-2-N_{\text {c } \max }\right)$ are the same. In this case, the cooperator candidate needs to compare the unreliability metrics of its parent source nodes $N_{\text {node }}-2-2 N_{\text {cmax }}$ times. In this way, the worst case happens when comparisons decreases from $N_{\text {node }}-2-N_{\text {cmax }}$ at the rate of $N_{\text {cmax }}$ a round until it becomes less than $N_{\text {cmax }}$. Therefore, its computational complexity is calculated as

$$
\begin{aligned}
& C C_{4}^{c m p} \\
&=O\left(\left(N_{\text {node }}-2\right)\left(\left\lceil\frac{N_{\text {node }}-2}{N_{\text {cmax }}}-1\right\rceil\right)\right. \\
&\left.\quad-\left(N_{\text {node }}-2\right)\left(\left\lceil\frac{N_{\text {node }}-2}{N_{\text {cmax }}}\right\rceil-1\right)\left\lceil\frac{N_{\text {node }}-2}{N_{\text {cmax }}}\right\rceil N_{\text {cmax }} / 2\right) \\
& \propto O\left(\left\lceil\frac{N_{\text {node }}}{N_{\text {cmax }}}\right\rceil N_{\text {node }}^{2}\right)
\end{aligned}
$$

where $\lceil(\cdot)\rceil$ is ceiling of $(\cdot)$, namely, the smallest integer not less than $(\cdot)$. Note this cost is still less than $O\left(N_{\text {node }}^{3}\right)$.

Consequently, the total computational complexity of Algorithm is given by the sum of these costs, and it is dominated by the largest complexity as

$$
C C_{4}=C C_{4}^{\text {algo3 }}+C C_{4}^{C}+C C_{4}^{S}+C C_{4}^{c m p} \propto O\left(N_{\text {node }}^{3}\right) .
$$

\section{Appendix 5}

For each $k\left(k=1,2, \cdots, N_{\text {node }}\right)$, we need to find the minimum out of a set of $M_{k, i}^{B A N C O}$ with the number of elements $=N_{\text {node }}-1$, so the computational complexity of the proposed BANCO selection is given by

$$
C C_{B A N C O}=O\left(\left(N_{\text {node }}-1\right) \times N_{\text {node }}\right) \propto O\left(N_{\text {node }}^{2}\right) .
$$

\section{Competing interests}

The authors declare that they have no competing interests.

\section{Acknowledgements}

This work was also supported in part by the Research Grant of Support Center for Advanced Telecommunications Technology Research (SCAT) of Japan.

\section{Author details}

${ }^{1}$ Department of Electronic Information System, Graduate School of Engineering, Osaka City University, 3-3-138, Sugimoto, Sumiyoshi, 558-8585 Osaka, Japan. ${ }^{2}$ Graduate School of Engineering, Osaka City University, 3-3-138 Sugimoto, Sumiyoshi, 558-8585 Osaka, Japan.

Received: 25 December 2014 Accepted: 6 October 2015

Published online: 29 October 2015 


\section{References}

1. IEEE Std.802.15.6-2012: IEEE standard for local and metropolitan area networks-Part 15.6: wireless body area networks

2. DB Smith, D Miniutti, TA Lamahewa, "Propagation models for body-area networks: a survey and new outlook". IEEE Antennas Propagat. Mag. 55(5), 97-117 (2013)

3. E Reusens, W Joseph, B Latre, B Braem, E Tanghe, L Martens, I Moerman, C Blondia, "Characterization of on-body communication channel and energy efficient topology design for wireless body area networks". IEEE Trans.Inform. Technol. Biomed. 13(3), 933-945 (2009)

4. A Fort, C Desset, P Wambacq, L Van Biesen, "An ultra-wideband body area propagation channel model-from statistics to implementation". IEEE Trans.Microwave Theory Tech. 54(4), 1820-1826 (2006)

5. Specification of the Bluetooth system covered core package version: 4.0(2010)

6. Zigbee health care profile specification. Revision 15, Version 1.0(2010)

7. A Sendonaris, E Erkip, B Aazhang, "User cooperation diversity.part i. system description". IEEE Trans. Commun. 51(11), 1927-1938 (2003)

8. JN Laneman, DNC Tse, GW Wornell, "Cooperative diversity in wireless networks: efficient protocols and outage behavior". IEEE Trans.Inform. Theory. 50(12), 3062-3080 (2004)

9. R D'Errico, R Rosini, M Maman, in Proc 2011 IEEE Int'l Conf. Commun. "A performance evaluation of cooperative schemes for on-body area networks based on measured time-variant channels", pp. 1-5

10. J Dong, D Smith, in Proc 2013 IEEE Int'l Conf. Commun. "Opportunistic relaying in wireless body area networks: coexistence performance", pp. 5613-5618

11. S Movassaghi, M Shirvanimoghaddam, M Abolhasan, in Proc 2013 Int'l Wireless Commun. Mobile Comput. Conf. A cooperative network coding approach to reliable wireless body area networks with demodulate-and-forward, pp. 394-399

12. DB Smith, D Miniutti, in Proc 2012 IEEE Wireless Commun. Networking Conf "Cooperative body-area-communications: first and second-order statistics with decode-and-forward", pp. 689-693

13. IEEE Std.802.15.4-2011: IEEE standard for local and metropolitan area networks-Part 15.4: low-rate wireless personal area networks (LR-WPANS)

14. M Maman, F Dehmas, R D’Errico, L Ouvry, in Proc 2009 IEEE Int'l Symp. Personal Indoor Mobile Commun. "Evaluating a tdma mac for body area networks using a space-time dependent channel model", pp. 2101-2105

15. P Abouzar, K Shafiee, DG Michelson, VCM Leung, in Proc. 2012-Spring IEEE Veh. Technol. Conf. "Effects of relaying on network lifetime in $2.4 \mathrm{GHz}$ IEEE802.15.4 based body area networks.", pp. 1-5

16. J Elias, A Mehaoua, in Proc 2012 IEEE Int'l Conf. Commun. "Energy-aware topology design for wireless body area networks," pp. 3409-3413

17. H Feng, B Liu, Z Yan, C Zhang, CW Chen, in Proc 2013 IEEE Int'l Symp. Personal Indoor Mobile Commun. "Prediction-based dynamic relay transmission scheme for wireless body area networks", pp. 2539-2544

18. J Ding, E Dutkiewicz, X Huang, G Fang, in Proc 2013 IEEE Int'l Conf. Ultra Wideband. "Energy-efficient cooperative relay selection for UWB based body area networks", pp. 97-102

19. S Hara, D Anzai, K Yanagihara, K Takizawa, K Hamaguchi, in Proc 2011 IEEE Int'l Symp. Personal Indoor Mobile Commun. "A cooperative transmission scheme for real-time data gathering in a wireless body area network", pp. 2254-2258

20. M Momoda, S Hara, in Proc 2013 Int'l Symp. Med. Inform. Commun. Technol. "A cooperative relaying scheme for real-time vital data gathering in a wearable wireless body area network", pp. 38-41

21. IEEE Std.802.15.4e-2012: IEEE standard for local and metropolitan area networks-Part 15.4: low-rate wireless personal area networks (LR-WPANs) amendment 1: MAC sublayer

22. M Momoda, S Hara, in Proc 2014 Int'l Symp. Med. Inform. Commun. Technol. "Use of IEEE 802.15.4e for a cooperator-assisted wireless body area network", pp. 1-5

23. E Rebeiz, G Caire, AF Molisch, "Energy-delay tradeoff and dynamic sleep switching for bluetooth-like body-area sensor networks". IEEE Trans Commun. 60(9), 2733-2746 (2012)

24. A Powerful System-On-Chip for 2.4-GHz IEEE 802.15.4, 6LoWPAN and ZigBee Applications, Texas Instruments

25. SG Akl, Parallel Sorting Algorithms. (Academic Press, Orlando, Florida, 1985)

\section{Submit your manuscript to a SpringerOpen ${ }^{\circ}$ journal and benefit from:}

- Convenient online submission

Rigorous peer review

- Immediate publication on acceptance

- Open access: articles freely available online

- High visibility within the field

- Retaining the copyright to your article

Submit your next manuscript at $\mathbf{s p r i n g e r o p e n . c o m ~}$ 